\title{
Spinous Process Osteotomy to Facilitate the Access to the Spinal Canal When Decompressing the Spinal Canal in Patients with Lumbar Spinal Stenosis
}

\author{
Erland Hermansen ${ }^{1,2}$, Gunnar Moen ${ }^{3}$, Anne Marie Fenstad ${ }^{4}$, Rune Birketvedt ${ }^{2}$, Kari Indrekvam ${ }^{2,5}$ \\ ${ }^{1}$ Department of Orthopedic Surgery, Ålesund Hospital, Møre and Romsdal Hospital Trust, Ålesund, Norway \\ ${ }^{2}$ Kysthospitalet in Hagevik,Orthopedic Clinic, Haukeland University Hospital, Bergen, Norway \\ ${ }^{3}$ Department of Radiology, Haukeland University Hospital, Bergen, Norway \\ ${ }^{4}$ The Norwegian Arthroplasty Register, Orthopedic Clinic, Haukeland University Hospital, Bergen, Norway \\ ${ }^{5}$ Department of Surgical Sciences, University of Bergen, Norway
}

\begin{abstract}
Study Design: Retrospective study.
Purpose: The main purpose of this study was to investigate the union-rate of the spinous process after performing a spinous process osteotomy and whether union affects the clinical results after surgery.

Overview of Literature: In the present study, spinous process osteotomy was used to facilitate access to the spinal canal when performing a decompressive procedure for lumbar spinal stenosis. The aim of this study was to evaluate the union rate of the spinous process and its effect on the clinical results of the procedure.

Methods: All patients were included in the study that underwent a decompressive procedure through spinous process osteotomy be between January 1, 2007 and December 31, 2007. Operation protocols were reviewed. A computed tomography (CT) scan was performed to evaluate the union of the osteotomies of the spinous process. According to the CT-scans, patients were divided into three groups: "complete-union," "partial-union," and "non-union." Patients reported their clinical results through a self-administered questionnaire.

Results: The mean period of follow up was 21.6 months (range, 16-28 months). A total of $44 \%$ of the performed osteotomies were considered as united. Ten patients (18\%) were classified as "complete-union," 30 patients $(55 \%)$ as "partial-union," and 15 patients (27\%) as "non-union." The "complete-union" group showed better clinical results and scored significantly better in the Oswestry Disability Index and EQ-5D. However, no statistical difference was found in the pain-scores. There were no differences between the "partial-union" group and the "no-union" group.

Conclusions: We found a radiologic union for 60 out of $135(44 \%)$ spinous process osteotomies.
\end{abstract}

Keywords: Spinal stenosis; Osteotomy

Received Nov 19, 2012; Revised Dec 19, 2012; Accepted Jan 9, 2013

Corresponding author: Erland Hermansen

Department of Orthopeadic Surgery, Aalesund Hospital,

Møre and Romsdal Hospital Trust, Ålesund, Norway

Tel: +47-70105766, Fax: +47-70105785, E-mail: erland.hermansen@helse-mr.no 


\section{Introduction}

Surgery for lumbar spinal stenosis (LSS) is the most common surgical procedure involving the adult lumbar spine [1].

LSS is defined as a narrowing of the spinal canal, with a reduction of the area of the dural sac and its contents. Stenosis develops from a bulging disc, hypertrophy of the facet-joints, and/or a hypertrophy of the ligamenta flava. Most commonly, a combination of all these three factors is present. This leads to neurogenic, claudiogenic pain and sometimes low back pain (LBP). The benefits of surgery for this condition is well documented, both in nonrandomized and randomized studies [2-5].

The surgical solution is to decompress the stenotic part of the lumbar spine. This means removal of the ligamenta flava, removal of the lamina (all or parts of it) along with partial medial facetectomy. Today, two main surgical approaches are used to achieve this decompression: laminectomy and fenestration procedures (multiple laminotomies). Both of these procedures have their pros and cons. Laminectomy-procedures are prone to postoperative instability [6], but gives wide decompression. Fenestrationprocedures are more technical demanding and does not provide as great a decompression $[7,8]$. In this study, spinous process osteotomy was used to facilitate access to the spinal canal. Spinous process osteotomy gives the surgeon the possibility to work across the midline, which facilitates the approach to the lateral recesses. This method is described by several authors [9-11], with good clinical results. Since the laminae are left mostly intact, this pro- cedure is considered to preserve stability, and one is able to perform a wide decompression. This gives an increase in the dural sac area that is close to normal values (Hermansen et al., in press), and is considerably higher than performing a laminotomi [7]. Thereby, the procedure has more potential advantages over the other methods. The surgical approach requires an osteotomy of the spinous process. If one level is operated on, two osteotomies are, whereas if two levels are operated on, three osteotomies are required, etc. After the procedure is completed, one expects spontaneous osseus or cartilaginous union of the osteotomy. The patient is mobilized without any restrictions. However, the exact rate of union, and the influence of this on the clinical results is not known $[9,11]$.

In other orthopaedic procedures, different osteotomies are used to facilitate exposure. These osteotomies may be associated with complications, for instance, in the form of pseudarthrosis and pain $[12,13]$. The main purpose of this retrospective study was to investigate the clinical results after performing a spinous process osteotomy. We wanted to examine the extent to which the osteotomy of the spinous process unites, and whether non-union was associated with clinical symptoms at follow-up.

\section{Materials and Methods}

The patient flow chart is shown in Fig. 1. A total of 177 lumbar decompression procedure were performed; of these, 73 patients underwent spinous process osteotomy. The decision of which surgical approach to use was made by surgeon preference, or based on the need for an addi-

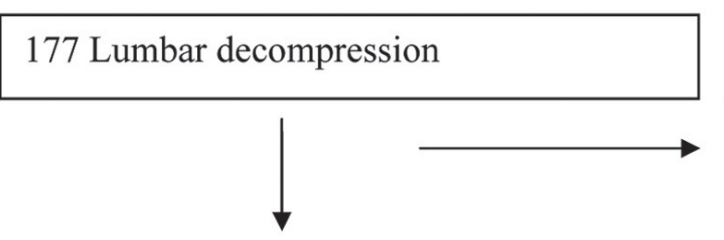

73 Spinous process osteotomy
81 Laminectomies, 9 fenestrationprocedures, 6 scar tissues release, 5 hemi-laminectomies and 3 wrong coded

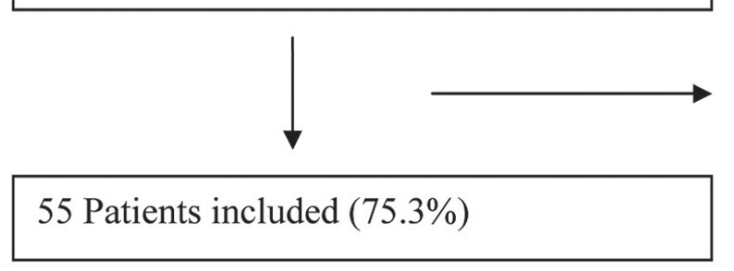

18 Excluded: Declined to attend: Cancer in the lungs: ALS: Later reoperated: Dead of unrelated causes: 2

Fig. 1. Flow chart of patient inclusion to the study, using spinous process osteotomy to facilitate acces to the spinal canal when decompressing the spinal canal in patients suffering from lumbar spinal stenosis. ALS, amyotrophic lateral sclerosis. 
tional procedure, such as fusion.

As a result, a total of 73 patients underwent spinous process osteotomy, and $55(75.3 \%)$ of these patients agreed to take part in the study. All patients had LSS without instability as judged by clinical and radiological methods.

The median age was 59 years (range, 36-87 years), and $56 \%$ of patients were male. Five (9\%) patients used nonsteroidal anti-inflammatory drug (NSAID) perioperatively. Nineteen (34\%) of the patients were smoking at the time of surgery. The mean number of levels decompressed was 1.76. Of the 55 patients, 8 (14\%) patients were operated at three levels, 26 (47\%) were operated at two levels, and 21 (38\%) were operated at one level. In total, 151 spinous processes were osteotomized in 55 patients.

The study patients were taken from the patient database, where we collected consecutively all the procedures that were registered as "ABC 56-Decompression of the lumbar spinal canal" and were carried out between January 1, 2007 and Desember 31, 2007 (Orbit 4.17; NCSP-code [NOMESCO Classification of Surgical Procedures]) [14].

Patients who had laminectomies, hemi-laminectomies and fenestration procedures were excluded.

1) Patient records were examined to identify all recorded perioperative and postoperative complications.
We noted whether the patients were using NSAIDs at the time of surgery and their smoking status.

2) A questionnaire was sent to the patients. The questionnaire consisted of functional ability scores, the Oswestry Disability Index (ODI), EQ-5D, and visual analogue score (VAS) scores for LBP and sciatic pain. The patients also did a self-evaluation of the effects of surgical treatment. The ODI-questionnaire was ODI Norwegian version 2.0 using a scale from 0 to 100 , in which lower values indicated better outcome. EQ-5D is a quality of life parameter, producing a scale from -0.59 to 1.00 , with higher values indicating better outcome. VAS pain-monitor was used to score LBP and sciatic pain in which the patients noted 0 for no pain and 10 for maximum pain. Both ODI and EQ-5D are reliable questionnaires and have been evaluated in several studies [15-19]. VAS-score is also recognised as a validated method of recording pain [20].

3) To assess the union of the spinous process osteotomy, computed tomography (CT)-scans with $3 \mathrm{~mm}$ slices and spacing of the operated level were done (GE HighSpeed 98). An experienced neuroradiologist performed the radiological evaluation. Full continuity of the spinous process was classified as "union," whereas discontinuity was classified as "non-union." The patients were divided
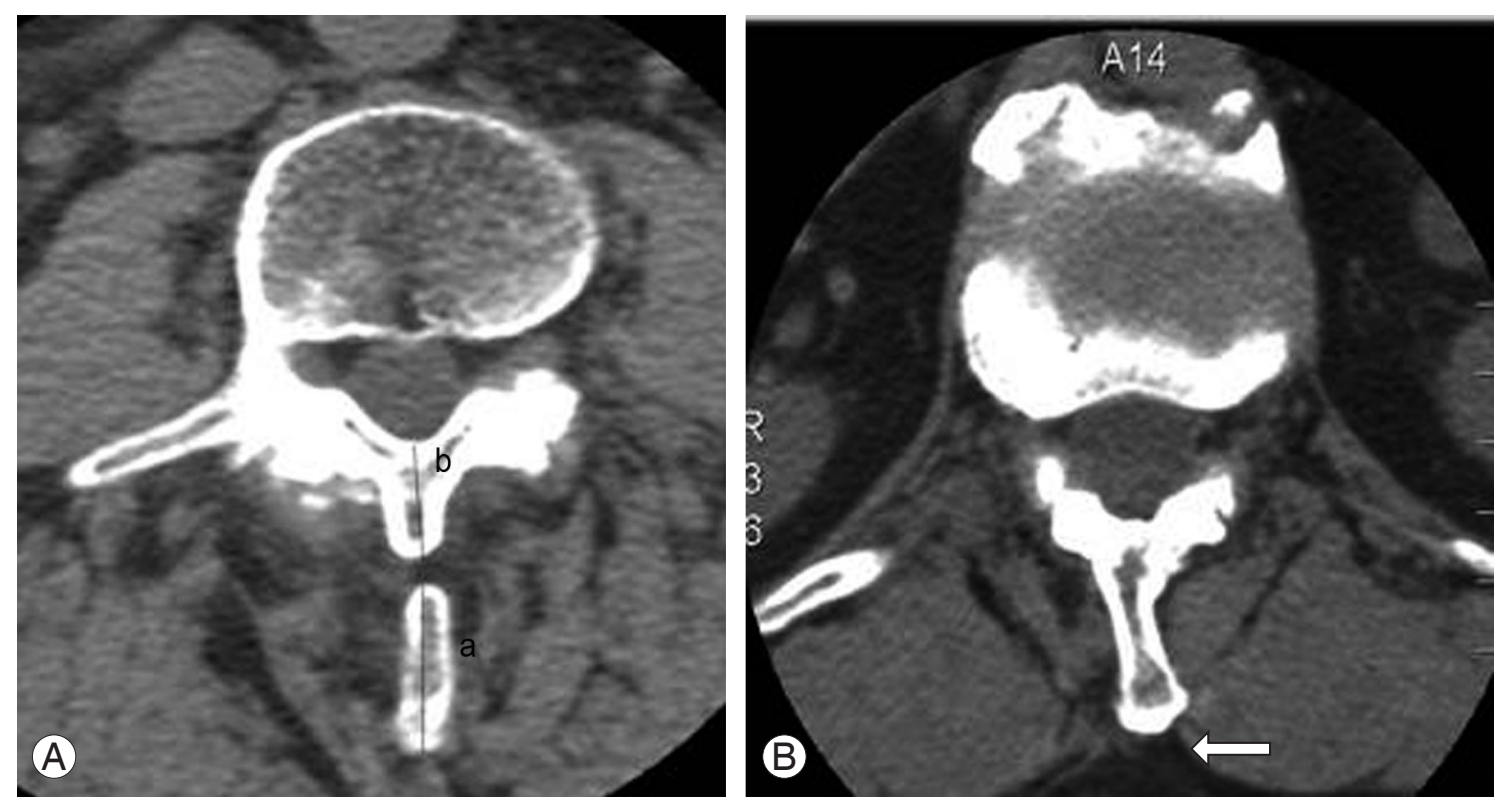

Fig. 2. (A) The spinous process ratio (osteotomi-basis coefficient) is calculated from the posterior part of spinous process divided on the basis and the anterior part of the remaining spinous process $(\mathrm{a} / \mathrm{b})$. This computed tomography (CT)-scan picture show a spinous process that is not united. (B) This picture show a CT-scan with a united spinous process. The arrow indicates were the osteotomy were performed. 
into three groups. The first group, "complete-union" had union of all the spinous processes. The second group, "partial-union" had union of some, but not all, of the spinous processes. The third group, "non-union" had no union of any of the spinous processes. We also measured the location of the osteotomy on the spinous process, and this was noted as a ratio between the posterior part versus the anterior part of the spinous process (Fig. 2). We called this the "spinous process ratio."

This study was approved by the Regional Committee for Medical and Health Research Ethics and the Norwegian Social Science Data Services (no. 21047).

All patients that agreed to join the study signed an informed consent paper.

\section{Surgical procedure}

The multifidus muscles were released unilaterally, and osteotomies were performed at the base of the spinous processes above and below the actual level. This provides a window between the two laminae that allows an adequate decompression from the midline access. Further options include flavectomy, partial laminotomy, and partial removal of the medial part of the facet joint. To decompress one level, about one third of the lower part of the upper lamina, and about one third of the upper part of the lower lamina were removed. Special attention was needed when performing decompression of two or three levels to keep the stability of the lumbar spine, and at least one third of the lamina was kept intact. To preserve a platform for the spinous process to reunite, a bridge of at least ten millimetres at the base of the spinous process was left intact. At the end of the procedure, the retractors were withdrawn, and the spinous processes with the intact supraspinal and interspinal ligaments were left in its original position without fixation. The patients were mobilized without any specific restrictions.

\section{Statistics}

All the results were analysed statistically with PASW Statistics ver. 18.0 for Windows (SPSS Inc., Chicago, IL, USA). The chi-square tests for the three union groups were performed for functional ability scores (ODI and EQ-5D), and VAS scores, self-reported effect of surgery, for smoking and use of NSAIDs. We also performed descriptive analysis with the mean, median and range.

Table 1. Complications identified during and after surgery with spinous process osteotomy $(n=73)$

Variable

Surgical complications

Dural tear

Cauda equine syndrome

Postoperative hematoma

Wrong-level operation

Superficial wound infection

Medical complications

\begin{tabular}{ll} 
Atrial fibrillation & $2(3)$ \\
\hline Urinary tract problem & $8(11)$ \\
Postoperative confusion & $1(1)$ \\
\hline
\end{tabular}

Table 2. Clinical and survey results during follow up of patients $(n=55)$

\begin{tabular}{lccc}
\multicolumn{1}{c}{ Variable } & Median & Mean & Range \\
ODI & 29 & 25.1 & $0-62$ \\
E0-5D & 0.74 & 0.74 & $0.23-0.98$ \\
VAS back-pain & 4 & 3.96 & $0-10$ \\
VAS leg-pain & 3 & 3.71 & $0-10$ \\
\hline
\end{tabular}

ODI, Oswestry Disability Index; VAS, visual analogue score.

\section{Results}

\section{Complications}

Perioperative complications are shown in Table 1. Following 73 procedures, there were eight (11\%) surgical complications. Some medical complications were also noted during the period of hospitalization, but none of these were life-threatening or disabling. Three patients (4\%) had a complication that required a second surgical procedure. The osteotomy of the spinous process created no surgical difficulties in managing the complications. Accidental dural tears were sutured without the need of additional removal of the lamina.

\section{Radiologic results}

We were able to classify union or non-union in 135 (89\%) out of 151 ostetomized spinous processes. Of these, CT scans showed radiological union in $60(44 \%)$ of 135 . Of the 55 patients examined radiologically, 10 (18\%) had "complete-union," 30 (55\%) had "partial-union," and 15 
Table 3. Clinical scores of patients undergoing surgery for lumbar spinal stenosis divided into three subgroups regarding union of the spinous processes

\begin{tabular}{lccc} 
& Variable & Complete-union & Partial-union \\
ODI & $10(0-30)^{\text {a) }}$ & $27(0-62)$ & $33(6-58)$ \\
EQ-5D & $0.90(0.7-0.98)^{b)}$ & $0.61(0.23-0.98)$ & $0.67(0.43-0.98)$ \\
VAS back-pain & $1.5(0-6)$ & $4.1(0-10)$ & $5.2(1-8)$ \\
VAS leg-pain & $1.9(0-7)$ & $3.6(0-10)$ & $5.1(0-8)$ \\
\hline
\end{tabular}

Values are presented as mean (range).

Complete-union, union of all spinous processes; Partial-union, union of some, but not all spinous processes; Non-union, union of none of the spinous processes; ODI, Oswestry Disability Index; VAS, visual analogue score.

${ }^{\text {a) }} p=0.002 ;{ }^{\text {b) }} p=0.007$.

(27\%) had "non-union." spinous process ratio was 2.37 (range, 0.5-6.2) in the spinous processes with union, and the ratio was 2.38 (range, $0.9-5.3$ ) in the spinous processes without union.

\section{Patient satisfaction}

The clinical results of all patients are listed in Table 2. All together, 40 (72\%) patients reported improvement, 5 (9\%) reported no improvement, and $10(18 \%)$ reported worsening of the condition (Fig. 3). The patients in the "complete-union" group scored better in the clinical questionnaires compared to the "partial-union" and "non-union" groups. This was statistically significant for ODI and EQ-5D. There were no statistically significant

\section{Histogram}

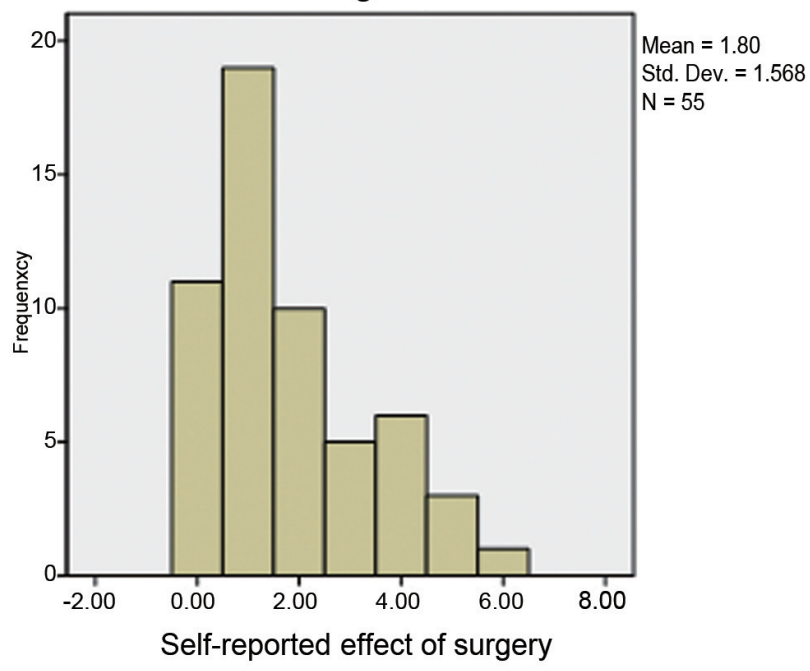

Fig. 3. Self-reported effect of surgery. 0, total improvement; 1, much improvement; 2, some improvement; 3 , no improvement; 4, some worse; 5 , much worse; 6 , worse than ever. differences in VAS-scores, smoking, use of NSAIDs, time for follow up, number of levels operated on, or gender between the three groups. There were no statistical differences between the "partial-union" group and the "nonunion" group (Table 3 ).

\section{Discussion}

LSS is a narrowing of the spinal canal leading to compression of nervous and vascular structures. The surgical solution is to relieve this compression by performing a decompressive procedure. To achieve this goal of decompression at the disc level where the narrowing primarily takes place, most parts of the ligamenta flava and the lamina (whole or parts of it) are removed. To decompress the lateral recesses, a partial medial facetectomy must be performed. In our previous study, we were able to restore the dural sac area to almost the normal values through spinous process osteotomy (Hermansen et al., in press). The same study also showed a positive correlation between increased dural sac area and patient satisfaction. Therefore, the most important factor in achieving good clinical results is to perform adequate decompression of the neural elements. Until now, laminectomy and fenestration-procedures have been the most frequently used methods to decompress the spinal canal. In this paper, spinous process osteotomy was used to facilitate accces to the spinal canal. By performing the osteotomy on the spinous process, one retains the advantages of both laminectomy and fenestration-procedures. Since the lamina is not totally removed and the supraspinal and interspinal ligaments are left intact, this surgical approach is theoretically stability preserving. This approach also gives a mid- 
line access that offers better visualisation, especially into the lateral recesses. However, it is not known whether the union of the osteotomy affects the clinical results, thus, the main aim of this study was to investigate whether non-union had a negative effect on the results.

The clinical results and patient satisfaction reported in the present study are good and comparable to other reports $[3,4,21]$. This was also shown in our previous study on the dural sac area before and after decompression (Hermansen et al., in press).

Also, the rate of complications are in agreement with other reports $[3,4,22]$.

Scapinelli et al. [23] showed that the orientation of the interspinal ligaments has an oblique orientation from anterior to posterior in a craniocaudal direction. Delank et al. [24] concluded in their cadaver study on spinal canal decompression that a bilateral hemilaminectomi did not result in increased motion. The supraspinal and interspinal ligaments in the cadavers were intact. Thus, even though a spinous process is not united, they keep their original orientation and location, and thereby the total stability of the spine should not be altered.

However, considering the clinical results between the three subgroups, we found that the "complete-union" group had better clinical results and scored significantly better in the functional ability scores (ODI and EQ-5D). No statistical difference was found in the pain-scores. It appears that "complete-union" (union of all the osteotomized spinous processes), gives better clinical results, but the number of "complete-union" patients is too small to draw a firm conclusion on this matter. However, the results in the present paper indicate that one should work to facilitate union. One important aspect is keeping a good bridge at the base of the spinous process. We tried to keep a base of at least ten mm, so that the spinous process has a firm bridge on which to re-unite. The measured spinous process ratio showed no difference between the "union" group versus the "non-union" group. However, we recommend that the osteotomy is placed near the base, since this will also facilitate the decompressive procedure. In Table 3, we noted that the VAS scores for "LBP" and "leg-pain" were different between the three groups. If non-union of the spinous process affects the results, one should expect that the difference in the VAS-scores is in "LBP" and not "leg-pain". Because the VAS-score for "legpain" should reflect the adequacy of the decompression of the neural elements.
Smoking and use of NSAIDs are factors known to impair bone-healing $[25,26]$. However, in this study, neither seemed to have an effect on the union on the spinous process. Nevertheless, in our sample, the number of patients using NSAIDs and the number of smokers were too small to provide any statistical basis for this conclusion.

This was a retrospective study with its limitations and is a descriptive analysis of the results. We have no baseline measurements of ODI or VAS scores to compare with the postoperative results.

We started using spinous process osteotomy as a method for decompressing the spinal canal with LSS in the autumn of 2006. One could expect to follow a learning curve initially, and hence, an improving rate of union over the first years.

\section{Conclusions}

CT-scan evaluation of the spinous process postoperatively after performing an osteotomy showed that the radiological union of the spinous process was found in 60 out of 135 (44\%). The clinical results seem to be better in patients that had union of all the spinous processes, and therefore, one should work to facilitate this union, when performing this procedure.

\section{Conflict of Interest}

No potential conflict of interest relevant to this article was reported.

\section{Acknowledgments}

We thank Paul Saunderson, MD, for help with the manuscript.

\section{References}

1. Ciol MA, Deyo RA, Howell E, Kreif S. An assessment of surgery for spinal stenosis: time trends, geographic variations, complications, and reoperations. J Am Geriatr Soc 1996;44:285-90.

2. Amundsen T, Weber H, Nordal HJ, Magnaes B, Abdelnoor M, Lilleas F. Lumbar spinal stenosis: conservative or surgical management? A prospective 10year study. Spine (Phila Pa 1976) 2000;25:1424-35.

3. Atlas SJ, Keller RB, Wu YA, Deyo RA, Singer DE. 
Long-term outcomes of surgical and nonsurgical management of lumbar spinal stenosis: 8 to 10 year results from the maine lumbar spine study. Spine (Phila Pa 1976) 2005;30:936-43.

4. Malmivaara A, Slatis P, Heliovaara M, et al. Surgical or nonoperative treatment for lumbar spinal stenosis? A randomized controlled trial. Spine (Phila Pa 1976) 2007;32:1-8.

5. Weinstein JN, Tosteson TD, Lurie JD, et al. Surgical versus nonsurgical therapy for lumbar spinal stenosis. N Engl J Med 2008;358:794-810.

6. Mullin BB, Rea GL, Irsik R, Catton M, Miner ME. The effect of postlaminectomy spinal instability on the outcome of lumbar spinal stenosis patients. J Spinal Disord 1996;9:107-16.

7. Dalgic A, Uckun O, Ergungor MF, et al. Comparison of unilateral hemilaminotomy and bilateral hemilaminotomy according to dural sac area in lumbar spinal stenosis. Minim Invasive Neurosurg 2010;53:60-4.

8. Subramaniam V, Chamberlain RH, Theodore N, et al. Biomechanical effects of laminoplasty versus laminectomy: stenosis and stability. Spine (Phila Pa 1976) 2009;34:E573-8.

9. El-Abed K, Barakat M, Ainscow D. Multilevel lumbar spinal stenosis decompression: midterm outcome using a modified hinge osteotomy technique. J Spinal Disord Tech 2011;24:376-80.

10. Gunzburg R, Keller TS, Szpalski M, Vandeputte K, Spratt KF. A prospective study on CT scan outcomes after conservative decompression surgery for lumbar spinal stenosis. J Spinal Disord Tech 2003;16:261-7.

11. Weiner BK, Fraser RD, Peterson M. Spinous process osteotomies to facilitate lumbar decompressive surgery. Spine (Phila Pa 1976) 1999;24:62-6.

12. Babhulkar S, Babhulkar S. Controversies in the management of intra-articular fractures of distal humerus in adults. Indian J Orthop 2011;45:216-25.

13. Glassman AH. Complications of trochanteric osteotomy. Orthop Clin North Am 1992;23:321-33.

14. Klassifikasjon av medisinske prosedyrer og kirurgiske inngrep 2010. Oslo: Sosial-og helsedirektoratet; 2010.

15. Davidson M, Keating JL. A comparison of five low back disability questionnaires: reliability and responsiveness. Phys Ther 2002;82:8-24.

16. Fairbank JC, Pynsent PB. The Oswestry Disability Index. Spine (Phila Pa 1976) 2000;25:2940-52.

17. Hansson T, Hansson E, Malchau H. Utility of spine surgery: a comparison of common elective orthopaedic surgical procedures. Spine (Phila Pa 1976) 2008;33:2819-30.

18. Jansson KA, Nemeth G, Granath F, Jonsson B, Blomqvist P. Health-related quality of life (EQ-5D) before and one year after surgery for lumbar spinal stenosis. J Bone Joint Surg Br 2009;91:210-6.

19. Solberg TK, Olsen JA, Ingebrigtsen T, Hofoss D, Nygaard OP. Health-related quality of life assessment by the EuroQol-5D can provide cost-utility data in the field of low-back surgery. Eur Spine J 2005;14:1000-7.

20. Ferreira-Valente MA, Pais-Ribeiro JL, Jensen MP. Validity of four pain intensity rating scales. Pain 2011;152:2399-404.

21. Celik SE, Celik S, Goksu K, Kara A, Ince I. Microdecompressive laminatomy with a 5-year follow-up period for severe lumbar spinal stenosis. J Spinal Disord Tech 2010;23:229-35.

22. Tafazal SI, Sell PJ. Incidental durotomy in lumbar spine surgery: incidence and management. Eur Spine J 2005;14:287-90.

23. Scapinelli R, Stecco C, Pozzuoli A, Porzionato A, Macchi V, De Caro R. The lumbar interspinous ligaments in humans: anatomical study and review of the literature. Cells Tissues Organs 2006;183:1-11.

24. Delank KS, Gercek E, Kuhn S, et al. How does spinal canal decompression and dorsal stabilization affect segmental mobility? A biomechanical study. Arch Orthop Trauma Surg 2010;130:285-92.

25. Boursinos LA, Karachalios T, Poultsides L, Malizos KN. Do steroids, conventional non-steroidal antiinflammatory drugs and selective Cox-2 inhibitors adversely affect fracture healing? J Musculoskelet Neuronal Interact 2009;9:44-52.

26. Sloan A, Hussain I, Maqsood M, Eremin O, ElSheemy M. The effects of smoking on fracture healing. Surgeon 2010;8:111-6. 